$2012-12$

Implicit Social Learning in Relation to Autistic-Like Traits

Hudson, Matthew

http://hdl.handle.net/10026.1/17633

10.1007/s10803-012-1510-3

Journal of Autism and Developmental Disorders

Springer Science and Business Media LLC

All content in PEARL is protected by copyright law. Author manuscripts are made available in accordance with publisher policies. Please cite only the published version using the details provided on the item record or document. In the absence of an open licence (e.g. Creative Commons), permissions for further reuse of content should be sought from the publisher or author. 


\title{
Implicit Social Learning in Relation to Autistic-Like Traits
}

\author{
Matthew Hudson - Tanja C. W. Nijboer • \\ Tjeerd Jellema
}

(C) Springer Science+Business Media, LLC 2012

\begin{abstract}
We investigated if variation in autistic traits in the typically-developed population (using the Autismspectrum Quotient, AQ) influenced implicit learning of social information. In the learning phase, participants repeatedly observed two identities whose gaze and expression conveyed either a pro- or antisocial disposition. These identities were then employed in a gaze-cueing paradigm. Participants made speeded responses to a peripheral target that was spatially pre-cued by a nonpredictive gaze direction. The low AQ group $(n=50)$ showed a smaller gaze-cueing effect for the antisocial than for the prosocial identity. The high AQ group $(n=48)$ showed equivalent gaze-cueing for both identities. Others' intentions/dispositions can be learned implicitly and affect subsequent responses to their behavior. This ability is impaired with increasing levels of autistic traits.
\end{abstract}

Keywords Intention - Gaze direction - Autism spectrum . Emotional expression · Gaze cueing · Implicit learning

\footnotetext{
M. Hudson $(\varangle)$

School of Business, Universidad del Norte, Bloque G, Piso 4, Barranquilla, Atlantico, Colombia

e-mail: mhudson@uninorte.edu.co

T. C. W. Nijboer · T. Jellema

Department of Psychology, University of Hull, Cottingham

Road, Hull HU6 7RX, UK

e-mail: t.c.w.nijboer@uu.nl

T. Jellema

e-mail: t.jellema@hull.ac.uk

T. C. W. Nijboer

Psychologische Functieleer, Helmholtz Instituut, Universiteit Utrecht, Heidelberglaan 2, 3584 CS Utrecht, The Netherlands
}

\section{Introduction}

We are not as in control of our social judgments as we think we are. Often, implicit learning of social information occurs that affects our judgments of others, without us being aware of it. Implicit learning has attracted far less attention than its explicit counterpart, even though it is thought to be at least as ubiquitous as explicit learning and can dramatically influence our behavior and social perception (e.g. Lieberman et al. 2002; Satpute and Lieberman 2006). The implicit character is reflected in the fact that the learning process does not require any internal deliberate rehearsal or reasoning. It happens involuntarily, subconsciously, irrespective of the subject's intentions, and may or may not result in explicit knowledge. That is, implicitly learned information may affect one's attitudes without one realizing it, or one may realize the particular attitude but have no clue as to where it came from, as the learning itself happened subconsciously. Implicit social learning might contribute to the forming of social intuition (Lieberman 2000). One's intuition may suggest to take a certain course of action or to (dis-)trust someone, even though there is no obvious reason for doing so.

Two bodily cues that are especially relevant for inferring someone's pro- or antisocial disposition are facial expression and gaze direction. Emotional facial expressions are a potent source of information regarding the (non-)cooperative intentions of others. Specifically, the expressions of happiness and anger convey a prosocial and antisocial disposition, respectively, and have been found to influence decision making in cooperative exchanges (Schmidt and Cohn 2001; van Dijk et al. 2008). For example, smiling elicits attributions of generosity and cooperation (Mehu et al. 2007; Scharlemann et al. 2001), and those who do so are afforded greater degrees of trust and fairer allocations 
of resources in games requiring reciprocated cooperation (Brown and Moore 2002; Mehu et al. 2007; Scharlemann et al. 2001). Gaze direction is also relevant for learning about someone's anti- or prosocial disposition and to anticipate his or her future actions (Hudson and Jellema 2011; Hudson et al. 2009). Interactions between gaze and facial expression are especially relevant in this respect. Various studies show that the facial expression differs in meaning depending on whether the gaze is directed at or away from the observer. A direct gaze can increase the perceived attractiveness of a happy face (Jones et al. 2006), and enhance the speed of processing and perceived intensity of angry expressions (Adams and Kleck 2003, 2005; Hess et al. 2007; Sander et al. 2007).

\section{Distribution of Autistic-Like Traits in the General Population}

There are indications that distinct individual differences exist in the ability to implicitly learn about others' dispositions on the basis of social cues. In autism spectrum disorders (ASD) a marked deficiency was found in the involuntary and spontaneous extraction of the meaning of social cues (Jellema et al. 2009; Senju et al. 2009), which may lead to a compromised understanding of the intentions and beliefs underpinning others' actions (Baron-Cohen 1995). It has been suggested that autistic-like social deficiencies may be found throughout the entire population to differing degrees, without a clear boundary between normality and psychopathology, and with clinical levels of autism at the high end of this continuum (Baron-Cohen et al. 2001a; Constantino and Todd 2003).

The Autistic-spectrum Quotient (AQ) questionnaire is one of the measures that has been developed to gauge autistic-like traits in the population at large (Baron-Cohen et al. 2001a). It is a self-report questionnaire with a maximum score of 50 indicating the highest degree of autistic traits. It was originally designed to consist of five subscales (communication, social skills, imagination, attention to detail, attention switching). However, subsequent validation and factor analyses have suggested four (Stewart and Austin 2009) or three factors (Hurst et al. 2007). Nevertheless, there is a general consensus that the AQ contains at least three independent factors measuring socialness, attention to detail, and communication (Stewart and Austin 2009). A score of 32 may be indicative of clinical levels of autistic traits (Baron-Cohen et al. 2001a), and the AQ is able to correctly discriminate those with an ASD diagnosis from TD individuals with an accuracy of $83 \%$ (Woodbury-Smith et al. 2005). AQ scores in the normal population show associations with cognitive deficits/styles that are distinctive of individuals with ASD. For example, weak central coherence has been proposed as a general cognitive characteristic of ASD, in which there is a tendency for local over global processing (Happé 1999). Such a tendency is also associated with TD individuals who score high on the AQ (Grinter et al. 2009; Reed et al. 2011; Sutherland and Crewther 2010). Comparable deficits between those with ASD and TD individuals who score high on the AQ have also been found in the perception of social stimuli, such as subtle emotional states derived from the face and/or voice (Baron-Cohen et al. 2001c; Golan et al. 2007). These studies show that the more autistic traits an individual exhibits, the less able they are to identify another's mental state from facial and vocal cues. Scores on the AQ also correspond to activation of proposed neuropsychological mechanisms underpinning ASD. For example, the posterior superior temporal sulcus (pSTS) is a cortical region heavily implicated in the perception of social stimuli (Jellema and Perrett 2002) and has been proposed as a source of the impairments observed in ASD (e.g. Redcay 2008). Indeed, the activity of the pSTS during face to face conversation is negatively correlated with AQ scores (Suda et al. 2011), as is the volume of pSTS white matter (Von dem Hagen et al. 2011).

If TD individuals with relatively high levels of autisticlike traits indeed, to some extent, share social/cognitive deficits with individuals with ASD, then it may be possible to enhance our understanding of ASD by studying these abilities in TD individuals. Individuals with high-functioning autism have been shown to be adept at implicitly learning the behavior of other people if it is based on systematic and predictable rule based contingencies (e.g., a gaze direction that predicts the location of an object on $80 \%$ of occasions; Ristic et al. 2005). However, social information is founded more on the ability to interpret social cues in relation to oneself. It is therefore important to evaluate whether autistic traits are associated with an inability to implicitly learn social information, despite a seemingly intact ability to implicitly learn generalized rule based information.

\section{The Current Study}

The complexity and rapidity of social interactions often makes explicit reasoning and learning about social information implausible and unfeasible. It requires an implicit component that forms associations independently of conscious awareness and with minimal attentional resources. The overall aim of the current study was to investigate whether TD individuals implicitly learn or 'pick-up' the dispositions that other agents have towards them on the basis of specific combinations of facial expression and gaze direction cues. Of specific interest is whether sensitivity to this information and the ability to involuntarily learn about other's intentions on the basis of contingencies between 
social cues is uniformly distributed across the TD population or is subject to individual differences, specifically in traits associated with ASD. Demonstrating that the ability to implicitly pick up contingencies between social cues and to use this to infer other's mental states is related to the extent to which TD individuals possess autistic traits may provide a further understanding of the nature of the social deficits in ASD. The task consisted of an initial learning phase, which aimed to enable participants to learn the social disposition of two identities. This was immediately followed by a test phase, which provided an indirect measure of the extent of implicit learning that had occurred.

As the task is new we will provide here a description and the rationale for choosing this design. Participants first completed a learning task, which presented short video clips of two agents (A and B). During this phase participants should learn that each agent holds a different intention toward the observer. The facial expression of each agent gradually changed from happy to angry or vice versa, and the gaze direction gradually changed from direct to averted or vice versa. Whenever agent $\mathrm{A}$ began to smile her/his gaze moved in the direction of the observer, and when agent $\mathrm{A}$ began to express anger the direction of gaze moved away from the observer. Therefore the agent was looking at the observer when expressing happiness and looking away when expressing anger. This identity therefore conveyed an emotionally positive and prosocial intention toward the observer. Agent B showed the reverse pattern, whenever agent $B$ began to smile her/his gaze moved away from the observer, and when agent $B$ began to express anger the gaze moved toward the observer. Therefore the agent was looking at the observer when expressing anger and looking away when expressing happiness. This identity therefore conveyed an emotionally negative and antisocial intention toward the observer. Thus, despite both identities expressing anger and joy for exactly the same amount of time, and were looking toward the observer and away from the observer for exactly the same amount of time, the specific combination of social cues were different for the two identities. Repeated presentations of these specific combinations allowed the observer to automatically associate each identity with the specific combinations of gaze and expression, and therefore learn the specific dispositions of the agents. Participants completed an unrelated task thus ensuring that minimal attention was afforded to the specific gaze/expression combinations. The paradigm was designed to enable implicit learning, while excluding any possibilities for explicit learning.

In the subsequent test phase we used a gaze-cueing paradigm employing the two identities of the learning phase as an indirect measure of the extent to which participants had implicitly learned the agents' dispositions. In a typical gaze-cueing paradigm, the gaze direction of a centrally presented face is averted to the left or right. Next, a target appears either to the left or right of the agent to which participants respond as quickly as possible. Response times are faster when the gaze is directed toward the congruent visual hemifield in which the target appears than the incongruent visual hemifield, suggesting a reflexive and automatic orientation of visual spatial attention in the direction a person is looking. The time course of the gaze cueing effect can be measured by varying the delay between onset of the cue and onset of the target (stimulus onset asynchrony, SOA). The minimum SOA at which a gaze cueing effect is reliably observed is $100 \mathrm{~ms}$, whilst the longest is about $1000 \mathrm{~ms}$ (Friesen and Kingstone 2003; Ristic et al. 2005). The early onset of the effect is comparable to the involuntary/reflexive orienting of attention elicited in response to exogenous cues such as a peripheral transient. These effects are stimulus driven and exert a bottom-up effect on attention orienting. However, such exogenous cueing diminishes after SOAs of $300 \mathrm{~ms}$. The prolonged influence of gaze cues beyond this time course suggests that orienting of attention to an agent's gaze direction is also underpinned by voluntary top-down processes (Friesen et al. 2004). This is because such cues are endogenous and require interpretation of their meaning to be understood, in a similar way to other centrally presented cues such as arrows.

Importantly, the magnitude of the gaze cueing effect has been found to be mediated by factors such as stimulus identity (Deaner et al. 2007; Frischen and Tipper 2006) and the spatial predictability of the identity's gaze direction with respect to target location (Friesen et al. 2004; Kuhn and Kingstone 2009; Tipples 2008). It therefore seems highly plausible that the gaze cueing effect would also be sensitive to attributions of the pro/anti-social dispositions to the identities and is therefore an ideal measure of the extent to which participants had implicitly learnt the agent's intentions. The rationale is that participants are expected to be less cooperative towards agents who hold an antisocial disposition towards them, and consequently show a smaller cueing effect in response to the gaze direction of these agents, whereas the prosocial disposition will induce cooperation leading to an increased gaze-cueing effect. Thus, an implicit learning effect would be reflected in a significant difference in the magnitude of the gaze-cueing effect induced by prosocial and antisocial agents.

The crucial factor in this design is the fact that the influence of what is learnt in the learning phase is social in nature, in that it is based on the implicit attributions made regarding the agent's mental state and intentions. It is possible that an observer could differentiate between the 
agents, either implicitly or explicitly, based only on the visual appearance of the stimuli. However, this low level information is inherently meaningless with respect to target location in the test phase, and there is no reason that the purely visual differences themselves would influence responses. Only if the observer interpreted the differences in terms of the mental state of the agent (pro- or antisocial) would we expect an influence of identity in the test phase.

Furthermore, the test phase is an indirect measure of implicit learning. The gaze direction of both identities is equally (non)-predictive of target location, and so processing of stimulus identity is not necessary to complete the task. Any differences in the gaze-cueing effect can therefore be attributed to prior learning of social disposition. In the absence of a learning phase, there would be no a priori reason to assume that participants would treat the two identities differently. The research question was: does implicit social learning take place in our experimental paradigm, and if so, is there evidence for a variation in the implicit social learning ability within the TD population with lowest abilities near the high AQ end? We tested this question with two different SOAs, 300 and $800 \mathrm{~ms}$. This would reveal whether the effect of the identities' disposition was influencing the involuntary orienting of attention in response to the gaze direction or the voluntary interpretation of the gaze direction. That is, if an effect of identity is found at an SOA of $300 \mathrm{~ms}$, then this would suggest that the influence of learnt social dispositions is rapid and involuntarily. If an effect of identity is found only with an SOA of $800 \mathrm{~ms}$, then this would suggest that only the later voluntary response to gaze direction is influenced by learnt social dispositions.

\section{Method}

\section{Participants}

Participants $(n=105)$ were undergraduate students from a range of departments in the Natural sciences, Humanities and Social sciences, and participated for course credit or a fee. All had normal or corrected-to-normal vision, and provided written informed consent prior to the experiment. After applying selection criteria (see "Results" for details), 98 participants were included in the analysis (48 females, 50 males, mean age $=20.6$ years, $\mathrm{SD}=1.7$ years).

\section{Stimuli}

Stimuli were created using Poser 7 animation software (Curious Labs, Inc., Santa Cruz, CA, \& e frontier, Inc., Scotts Valley, CA). Two male characters and two female characters were used, though each participant was only exposed to two of them, and these were counterbalanced across participants. The agents were oriented facing the observer throughout the presentation and were depicted from the top of the shoulders upwards. The subtended angle of the agent's width varied from $11.8^{\circ}$ to $19.7^{\circ}$ (depending on the character used) whilst the height of all agents was $18.1^{\circ}$. The stimuli were presented on a 21 inch monitor $(100 \mathrm{~Hz}$ refresh rate) using e-Prime software (Psychology Software Tools, Inc., Sharpsburg, PA), at a distance of approximately $50 \mathrm{~cm}$ from the participant. A further description of the stimuli specific for the learning and test phases is given in the Procedure section below.

\section{Procedure and Task}

Prior to the experiment, participants completed an online version of the AQ (Baron-Cohen et al. 2001a) which took $10 \mathrm{~min}$ to complete. The experiment consisted of two phases: an initial implicit learning phase followed by a test phase (see Fig. 1). Written and verbal instructions were given prior to each phase. Participants were instructed that the experiment was about face perception, and were kept unaware of the real experimental aim.

\section{Learning Phase}

Each trial began with a blank screen of 1,500 ms duration, followed by the presentation of a video clip. Clips consisted of 20 frames, with the first frame on screen for $750 \mathrm{~ms}$ and the subsequent 18 frames for $50 \mathrm{~ms}$ each (1,650 ms total duration). The final frame remained on screen until a response (see below) was made or $3 \mathrm{~s}$ had elapsed. During the course of the clip, the agent's gaze direction was either initially fixated at the observer and then gradually averted horizontally until at a $30^{\circ}$ angle away from the participant by the end of the clip, or began with a $30^{\circ}$ aversion and ended directed at the observer. The gaze manipulation was accompanied by a change in emotional expression, which morphed either from a happy to an angry expression, or vice versa from an angry to a happy expression. Both gaze direction and emotional expression changed with a smooth and continuous motion during the course of the video clip. Specific combinations of the changes in gaze and expression were assigned to each of the two agents. For agent A, a gaze movement averting away from the observer was accompanied by a change from a happy to an angry expression, while a gaze movement toward the observer was accompanied by a change from angry to happy. Thus, whenever agent A was happy, attention was directed at the observer, and whenever agent A was angry attention was directed elsewhere, implying a prosocial disposition toward the observer. For the second identity (agent B), the reverse combinations were 
Learning phase: Prosocial agent

a

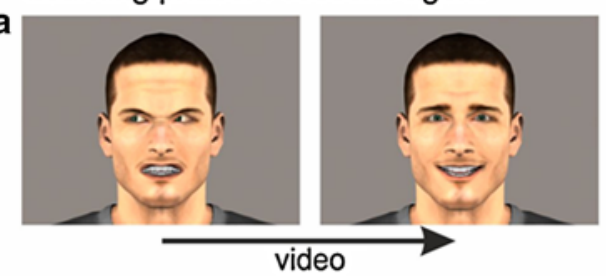

Learning phase: Antisocial agent

b

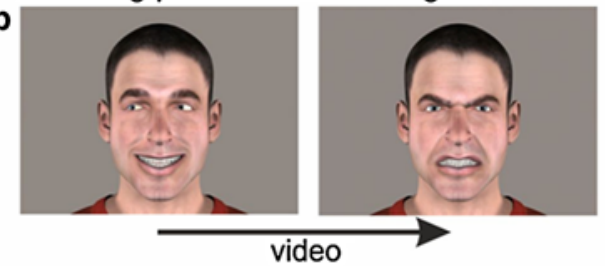

Test phase: Gaze-cueing paradigm

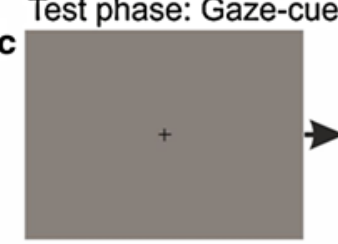

Fixation

$500 \mathrm{~ms}$

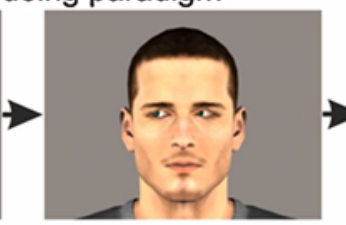

Gaze cue

$300-800 \mathrm{~ms}$

Fig. 1 Stimuli in the learning and test phases. Agents holding a prosocial disposition (a) and an antisocial disposition (b) toward the observer. For all identities the gaze changed gradually from averted to directed at the observer (left panels) or from direct to averted (right

presented, with a change in facial expression from angry to happy coinciding with a gaze movement away from the observer, and a change from happy to angry coinciding with a gaze movement toward the observer. These combinations of gaze and expression implied an antisocial disposition toward the observer, as the angry expression was directed at the observer, while the happy expression was directed away.

An important feature of this design was that each agent displayed angry and happy expressions, and gaze directed at the observer and away from the observer, for exactly the same number of times and for the same duration. This was important because we specifically aimed to test the ability for implicit social learning by having the stimuli as similar as possible to one another with only subtle differences, and thus wanted to exclude any possibilities for explicit learning. Further, by using the films also in reversed order an effect for the most recently presented frame (i.e. the frame the clip ended with) was excluded, as each agent's action ended in a happy and an angry expression, and a direct and averted gaze, the same number of times. Debriefing of participants at the conclusion of the experiment confirmed that they did not realize that one identity was antisocial and the other prosocial.

During the learning phase $(15 \mathrm{~min})$ the participants observed 64 of these video-clips, 32 for each the two
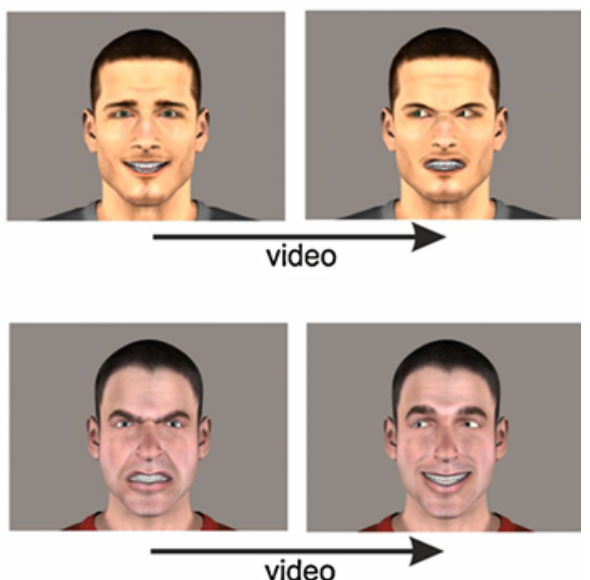

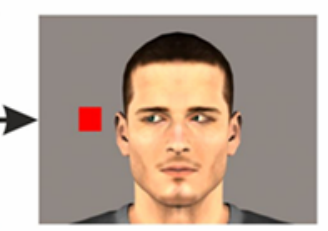

Target valid

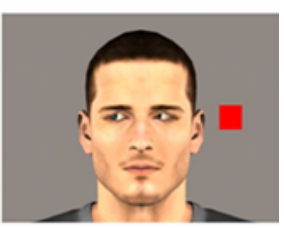

Target invalid panels). Only the first and last frames of each video are shown. $\mathbf{c}$ The trial procedure for the test phase, in which the onset of the gaze cue preceded the onset of a target at the gazed at (valid) or opposite location (invalid) location by 300 or $800 \mathrm{~ms}$

identities (randomly presented). The left/right aversion of the gaze was counterbalanced across trials. To ensure participants paid attention to the stimuli throughout the duration of the clips, a task was introduced, which required participants to press the spacebar when the gaze stopped moving near the end of each trial. To prevent participants from simply pressing the spacebar without paying attention to the stimuli, sixteen catch trials were added (eight for each identity) in which the gaze remained fixed at $0^{\circ}$ (bringing the total number of trials to 80) and in which no response was required. In the catch trials the facial expression remained neutral so as not to interfere with the implicit learning. Erroneous responses consisted therefore of the absence of a response in those trials where the eyes moved and the presence of a response when the eyes remained motionless.

\section{Test Phase}

The test phase consisted of a gaze-cueing paradigm involving the two identities previously observed in the learning phase. After a fixation cross $(500 \mathrm{~ms})$, a face was presented centrally with gaze averted towards either the left or right of the screen (Fig. 1c). The size and orientation of the face and shoulders were the same as in the learning phase, but the facial expression of the agents was neutral 
throughout. The extent of gaze aversion was fixed at $30^{\circ}$ from mutual gaze. After an SOA of either 300 or $800 \mathrm{~ms}$, a peripheral target (red square) appeared in a location either congruent (valid) or incongruent (invalid) with the agent's gaze direction. The square's height and width was $2.1^{\circ}$; its inner edge was positioned $6.9^{\circ}$ from the centre of the screen and either $1.3^{\circ}$ and $0.9^{\circ}$ from the edge of the face stimulus (depending on the character used). The gaze cue was non-predictive for the target's location. Participants completed 96 trials (48 per identity). Participants were required to press the spacebar as quickly as they could upon detection of the target. To ensure participants were indeed responding when they detected the target and not indiscriminately pressing the spacebar on each trial, 32 catch trials (16 per identity) were included in which the target did not appear and no response was required. Erroneous responses consisted therefore of the absence of a response in trials when the target was present and the presence of a response in trials when the target was absent. The duration of the test phase was approximately $5 \mathrm{~min}$. The SOA of 300 reflected the optimal timing for reflexive exogenous gaze-cuing effects (typically between 150 and $450 \mathrm{~ms}$ ), while at $800 \mathrm{~ms}$ gaze cueing would be governed by voluntary endogenous processes.

\section{Results}

The error rates during the learning phase (mean $=3.0 \%$, $\mathrm{SD}=11.0 \%)$ and during the test phase (mean $=0.5 \%$, $\mathrm{SD}=1.1 \%) \quad$ were averaged $\quad($ mean $=1.7 \%, \quad \mathrm{SD}=$ $5.6 \%)$. Participants with an overall error rate exceeding $10 \%$ were excluded $(n=7$, mean $=18.0 \%, \mathrm{SD}=13.4 \%)$, leaving 98 participants (mean $=0.6 \%, \mathrm{SD}=1.3 \%$ ) in the analysis. Response anticipations $(<100 \mathrm{~ms})$ and outliers ( $>2 \mathrm{SD}$ above each participants mean RT) were excluded $(4.4 \%)$.

The mean AQ score was 15.3 ( $\mathrm{SD}=4.6)$. Participants scoring less than the median AQ score of 16 were allocated to the Low AQ group $(n=50$, mean $=11.5, \mathrm{SD}=2.5)$, while those scoring 16 or above were allocated to the High AQ group $(n=48$, mean $=19.1, \mathrm{SD}=2.7)$.

A $2 \times 2 \times 2 \times 2$ ANOVA was conducted on the mean reaction times (RT) with SOA (300 vs. $800 \mathrm{~ms}$ ), Disposition (prosocial vs. antisocial) and Cue-target validity (valid vs. invalid) as within subjects factors, and AQ group (low vs. high) as a between subjects factor (see Fig. 2). There was a significant main effect of $\operatorname{SOA}(F(1,96)=8.0$, $\left.p=.006, \eta_{p}^{2}=0.077\right)$, with RTs faster when the SOA was $800 \mathrm{~ms} \quad($ mean $=329.8 \mathrm{~ms} \quad \mathrm{SD}=41.1 \mathrm{~ms})$ than when $300 \mathrm{~ms}$ (mean $=336.2 \mathrm{~ms}, \mathrm{SD}=42.3 \mathrm{~ms}$ ). This reflected a foreperiod effect whereby the increased SOA enhances the participant's preparation for the upcoming target. The main effect of Cue-target validity approached significance $\left(F(1,96)=3.74, p=.056, \eta_{p}^{2}=0.037\right)$, with RTs marginally faster when cue and target were valid (mean $=331.8 \mathrm{~ms}, \quad \mathrm{SD}=40.1 \mathrm{~ms}$ ) than when invalid (mean $=334.2 \mathrm{~ms}, \mathrm{SD}=41.2 \mathrm{~ms}$ ). There was a significant main effect of AQ group $(F(1,96)=4.69, p=.033$, $\left.\eta_{p}^{2}=0.047\right)$, with the High AQ group responding quicker (mean $=324.2 \mathrm{~ms}, \mathrm{SD}=30.3 \mathrm{~ms}$ ) than the Low AQ group (mean $=341.5 \mathrm{~ms}, S D=53.2 \mathrm{~ms}$ ). There was no main effect of Disposition $(F(1,96)=0.385, p=.536$, $\left.\eta_{p}^{2}=0.004\right)$. None of the two-way or three-way interactions were significant (all $p$ 's $>.05$ ). Importantly though, the four-way interaction was significant $(F(1,96)=5.14$, $p=.026, \eta_{p}^{2}=0.051$ ). To investigate the four-way interaction further, the magnitude of the cueing effect $\left(\mathrm{RT}_{\text {diff }}=\mathrm{RT}_{\text {invalid }}-\mathrm{RT}_{\text {valid }}\right)$ was entered into two separate 2-way ANOVAs (Disposition $\times$ AQ group), one for $300 \mathrm{~ms}$ SOA and one for $800 \mathrm{~ms}$ SOA (for each $\alpha$ was adjusted at 0.025 ).

At a SOA of $300 \mathrm{~ms}$ (Fig. 2a), there was no main effect of Disposition $\left(F(1,96)=1.19, p=.278, \eta_{p}^{2}=0.012\right)$ and no main effect of group $(F(1,96)=3.31, p=.072$, $\left.\eta_{p}^{2}=0.033\right)$. There was, however, a significant two-way interaction $\left(F(1,96)=5.3, p=.024, \eta_{p}^{2}=0.052\right)$. The Low AQ group showed a significantly smaller gaze cueing effect in response to the antisocial identity (mean = $-4.0 \mathrm{~ms}, \mathrm{SD}=21.8 \mathrm{~ms}$ ) compared to the prosocial identity $($ mean $=5.5 \mathrm{~ms}, \mathrm{SD}=24.2 \mathrm{~ms}, t(49)=2.62, p=.012)$. In contrast, the gaze cueing effect exhibited by the High AQ group did not differ between the two identities (antisocial: mean $=9.5 \mathrm{~ms}, \mathrm{SD}=23.6 \mathrm{~ms}$, prosocial: mean $=6.1 \mathrm{~ms}$, $\mathrm{SD}=25.5 \mathrm{~ms}, t(47)=-0.789, p=.434) .{ }^{1}$ Further, the cueing effects of low and high AQ groups were identical for the prosocial identity $(t(96)=-0.131, p=.896)$, but differed for the antisocial identity $(t(96)=-2.96, p=.004)$.

The same analysis conducted for the $800 \mathrm{~ms}$ SOA condition (Fig. 2b) revealed no significant main effects of Disposition $\left(F(1,96)=0.005, p=.945, \eta_{p}^{2}=0.000\right)$ or AQ group $\left(F(1,96)=0.33, p=.567, \eta_{p}^{2}=0.003\right)$, and no significant interaction $(F(1,96)=1.51, \quad p=.221$, $\left.\eta_{p}^{2}=0.016\right)$.

It should be noted that the Low AQ group $(n=50)$ contained more females $(n=28)$ than males $(n=22)$ whereas the High AQ group $(n=48)$ contained more males $(n=28)$

\footnotetext{
${ }^{1}$ As we employed a median split to create the low and high AQ groups, the mean AQ score for the high AQ group was low compared to the threshold of 32 for clinical levels of autistic traits (Baron-Cohen et al. 2001a). The data was therefore re-analyzed with the High AQ group defined as those in the upper quartile of AQ scores, with an AQ score of 19 or more $(n=24, M=21.3, \mathrm{SD}=1.9)$. However, the results following this manipulation were no different from those using the median split $(300 \mathrm{~ms}$ SOA: $t(23)=0.427, p=.673 ; 800 \mathrm{~ms}$ SOA: $t(23)=0.772, p=.448)$.
} 
Fig. 2 Gaze cueing in response to the pro- and antisocial identities with SOAs of $300 \mathrm{~ms}$ (a) and $800 \mathrm{~ms}$ (b) for the Low and High AQ groups. The righthand panels in (a) and (b) show a comparison between the Low and High AQ groups of the magnitude of the gaze cueing effect $\left(\mathrm{RT}_{\text {invalid }}-\mathrm{RT}_{\text {valid }}\right)$ elicited by the prosocial and antisocial identities
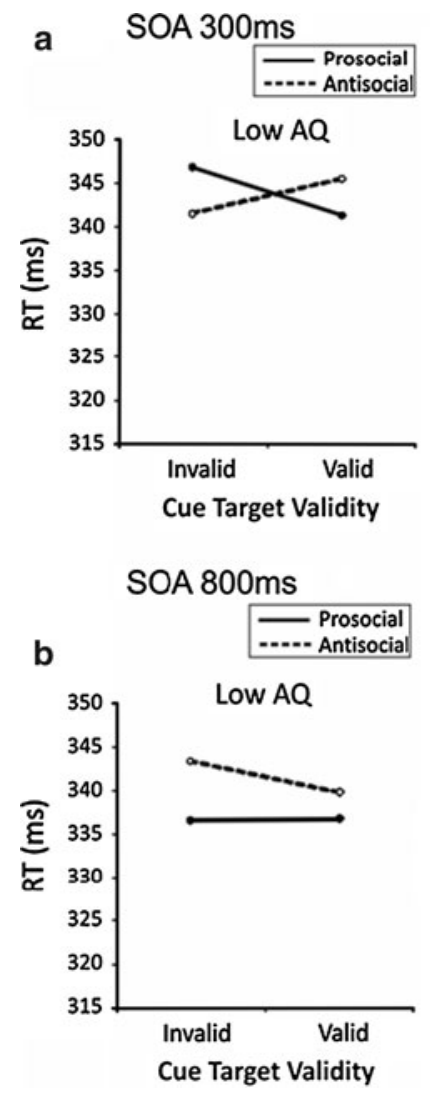

than females $(n=20)$. To see whether the difference between low and high AQ groups found at $300 \mathrm{~ms} \mathrm{SOA}$ might have been related to these different gender ratios, we performed the same $2 \times 2$ ANOVA on $\mathrm{RT}_{\text {diff }}$ with Disposition as the within subjects factor but now with Gender as the between-subjects variable. This showed no main effects of Disposition $\left(F(1,96)=1.19, p=.278, \eta_{p}^{2}=0.012\right)$ or Gender $\left(F(1,96)=1.22, p=.273, \eta_{p}^{2}=0.013\right)$ and no interaction $\left(F(1,96)=1.96, p=.165, \eta_{p}^{2}=0.02\right)$, indicating that Gender could not explain the difference in cueing effect between the AQ groups.

\section{Correlations}

To further investigate the relationship between autistic traits and the ability for implicit social learning, we correlated the cueing effect $\left(\mathrm{RT}_{\text {diff }}\right)$ elicited by the anti- and prosocial agents with scores on the AQ (Pearson, $n=98$, two-tailed). At $300 \mathrm{~ms} \mathrm{SOA}$, the cueing effect elicited by the prosocial identity showed no significant correlation with scores on the AQ $(r=0.082, p=.424)$. However, the cueing effect elicited by the antisocial identity was positively correlated with AQ scores $(r=0.258, p=.01)$. At $800 \mathrm{~ms}$ SOA there were no significant correlations for the prosocial identity (all $p$ 's $>.245$ ) or the antisocial identity (all $p$ 's $>$.137).
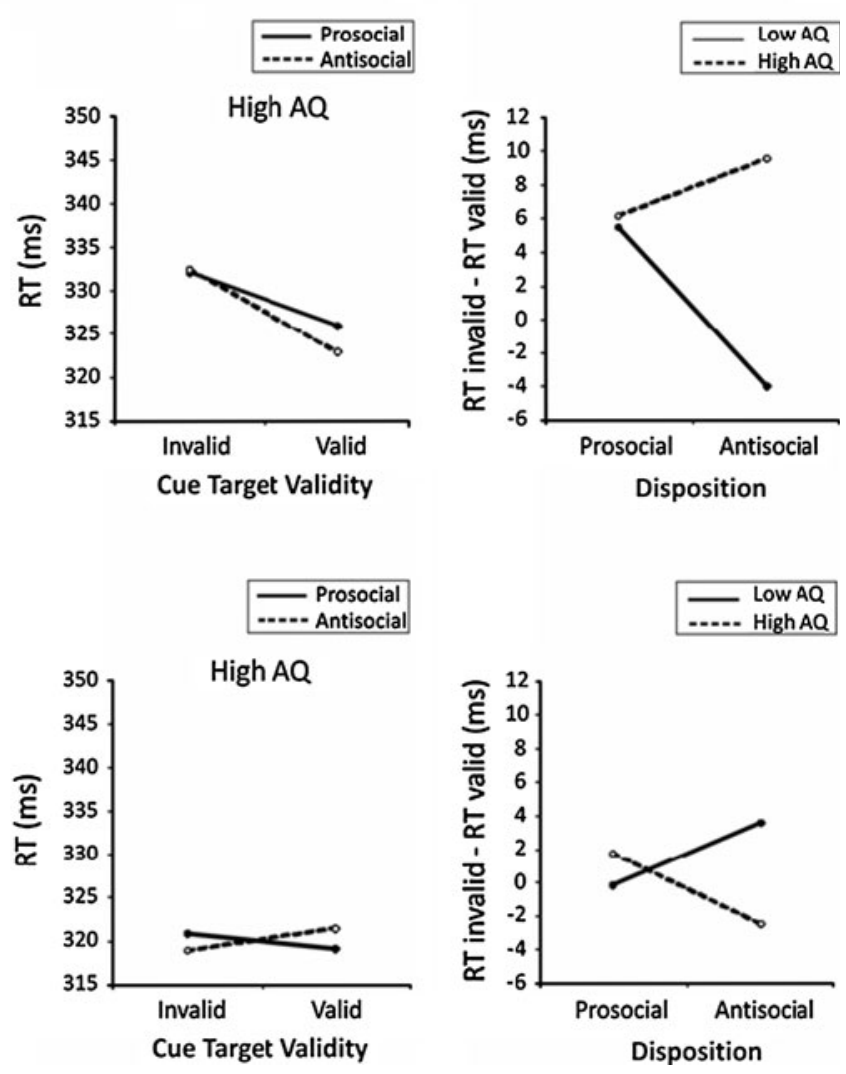

\section{Discussion}

We examined whether TD individuals implicitly learned the dispositions other agents held towards them (as conveyed by specific combinations of facial cues), and whether this capacity was related to the extent of autistic-like traits measured with the AQ. We found evidence for implicit social learning, but only in the Low AQ group. With an SOA of $300 \mathrm{~ms}$, the gaze-cueing effect of the Low AQ group elicited by the antisocial identity was significantly smaller than that elicited by the prosocial identity, and was significantly smaller than the gaze-cueing effects in response to either identity by the High AQ group. Furthermore, this reduction in gaze-cueing became larger as scores on the AQ decreased. With an SOA of $800 \mathrm{~ms}$, there was no influence of $\mathrm{AQ}$ on the gaze cueing effects in either the group or correlation analyses.

Thus, modulation of the cueing effect was only evident in the short SOA condition, which is the result of involuntary exogenous orienting, but not in the longer SOA condition, which reflects voluntary endogenous orienting (Friesen et al. 2004). This suggests that implicit social learning affects the earliest responses to another's gaze direction, in line with findings that personal familiarity can affect gaze-cueing at comparable time courses (Deaner et al. 2007). In contrast, an influence of the spatial predictability of the gaze cue only 
affects later orienting responses (Bayliss et al. 2009; Bayliss and Tipper 2006). This may suggest a possible dissociable effect of social and non-social information on gaze-cueing, although further research is necessary to corroborate this. A female advantage in gaze-cueing tasks has been reported (Bayliss et al. 2005) and there were more females than males in the Low AQ group and more males than females in the High AQ group. However, participant gender was not found to yield any significant differences on the gaze-cueing task, and so could not explain the difference between the low and high AQ groups.

We posit that the learning phase caused the discrepancy between the results of the low and high AQ groups. That is, the Low AQ group implicitly learned the social dispositions whereas the High AQ group did not. The task in the learning phase did not necessitate explicit processing of the identity-specific gaze/expression contingencies and the participants were unaware that they would encounter these identities again in the test phase. Therefore, the participants had no motivation to learn the gaze/expression contingencies, and indeed debriefing of participants afterwards revealed they had not noticed that each identity had an idiosyncratic pattern of gaze and expression behavior. This suggests that these attributions were automatic and implicit, without the participants being aware of it. These involuntary attributions then manifested themselves in the response to the gaze direction of the identity in the cueing paradigm. The results thus suggest an automatic influence of identity on reflexive gaze-cueing.

However, we should also consider whether the effects found in the cueing task could be explained by factors other than implicit social learning. In principle it could be that specific characteristics of the low and high AQ groups, unrelated to a capacity for implicit social learning, caused the discrepancy in the cueing effects in response to the two identities. It is possible that the Low AQ group showed increased sensitivity to the size of the eye region or to the identity's familiarity, factors which have previously been shown to mediate gaze-cueing effects (eye region, Tipples 2005; familiarity, Deaner et al. 2007). However, the specific identities used for the pro- and anti-social conditions were counterbalanced across participants, and each identity was presented for an equal amount of time, preventing an effect of familiarity. Further, a tendency to overestimate the presentation frequency of negative emotional expressions has been previously demonstrated (Johansson et al. 2004), which may have caused participants to subjectively remember the antisocial identity as being more familiar. However, familiarity has been shown to enhance the gazecueing effect and so cannot account for the reduction observed for the antisocial identity in the Low AQ group.

With respect to the high AQ group, several factors might in principle have contributed. Firstly, it could be argued that they may have been mildly impaired in expression recognition, limiting their ability to read the intentions of the agents from their expressions. However, only maximally intense happy and angry expressions were used, which are flawlessly recognized even by individuals with high-functioning ASD (Golan et al. 2007). Secondly, individuals with ASD exhibit weak central coherence (Happé 1999), involving an impaired ability to integrate features into a coherent percept. If the high AQ group would also have suffered to some extent from weak central coherence, then it might have prevented them from integrating the gaze and expression, which would have impeded their ability to infer which identity was prosocial and which was antisocial. However, other studies have found that integration of eye gaze and facial attributes (e.g. head movement; Hudson et al. 2011) is evident in those with ASD and so the same can be expected of those with high AQ scores. Thirdly, a more generalized impairment in perceptual learning, not specific to social stimuli, may have caused the lack of an identity effect in the High AQ group. There is some evidence that individuals with ASD do not extract relevant information from repeated exposure to visual patterns as readily as TD individuals (Plaisted et al. 1998), and that those with high AQ scores outperform those with low AQ scores on these tasks (Reed et al. 2011). To control for a more general perceptual learning deficit, future studies could contrast social and non-social learning conditions.

A further finding of interest is that the High AQ group exhibited faster overall RTs than the Low AQ group. This agrees with previous studies investigating the effect of autistic traits on gaze-cueing, which show that those with ASD respond quicker overall than TD individuals (Chawarska et al. 2003; Vlamings et al. 2005). This has been proposed to reflect that in TD individuals, the gaze direction of another person is afforded additional processing that enables the observer to interpret the intentions and mental state of the gazer, whereas this additional processing is reduced in those with ASD (Driver et al. 1999; Nation and Penny 2008; Ristic et al. 2005). The current study shows that the effect of autistic traits on RTs in gazecueing may also be evident in the TD population. However, another possibility is that more general group differences, such as in IQ or motor performance, played a role. As no such data was collected in the current study, we cannot substantiate this, which is a limitation of the study.

There was only a marginal main effect of cue-target validity. That is, the overall influence of gaze direction on attention orienting was small, which is surprising given the robust nature of this effect (Driver et al. 1999: Langton and Bruce 1999). This could partly be attributable to the small (near negative) cueing effect in response to the antisocial identity by the Low AQ group (i.e. shorter RTs for invalid 
cueing than for valid cueing). Another contributing factor may have been the distinct lack of a gaze-cueing effect at $800 \mathrm{~ms}$ SOA. This suggests that the current method may not have been optimal in eliciting a gaze cueing effect. However, this does not detract from the main finding of the study.

A further implication of these results is that implicit learning of pro- and antisocial dispositions are not equally effective. Implicit learning occurred exclusively for the antisocial identity which caused a reduction in the gazecueing effect, whereas no modulation was observed for the prosocial identity. This may be indicative of a biased sensitivity to detecting potential untrustworthiness in others rather than trustworthiness. This notion is supported by a range of studies. For example, observers can discriminate and recall faces of those who defect on another's cooperation and who are therefore considered to be untrustworthy better than those who reciprocate another's cooperation (Bayliss and Tipper 2006; Brown and Moore 2002; Verplaetse et al. 2007; Yamagishi et al. 2003), and that the faces of those defecting on another's cooperation capture visual spatial attention (Vanneste et al. 2007). Therefore, the effect obtained for the antisocial identity but not the prosocial identity may reflect a greater tendency to avoid individuals who may incur a cost in social interactions than to promote engagement with those whose interactions may be beneficial.

It has been reported that in the TD population a smaller gaze-cueing effect is associated with higher scores on the AQ (Bayliss and Tipper 2005). It could be argued that this finding renders the gaze-cueing task unsuitable as a measure of implicit social learning, as there would be a low AQ versus high AQ difference anyway, irrespective of whether implicit learning had taken place. However, several studies indicate that even individuals with ASD, who occupy a position at the extreme high end of the autistic-trait dimension (Baron-Cohen 1995, 2005), show reflexive orienting to non-predictable gaze cues (Chawarska et al. 2003; Kemner et al. 2006; Kylliainen and Hietanen 2004; Senju et al. 2004; Swettenham et al. 2003; Vlamings et al. 2005). Only one study has found this not to be the case (Ristic et al. 2005). The wealth of evidence is in favor of individuals with ASD showing reflexive orienting to nonpredictive gaze cues, and one would therefore expect TD individuals with high scores on the AQ to also show intact reflexive gaze-cueing. Indeed, not only did our results support this conclusion, but in the case of the antisocial identity, we found a greater gaze-cueing effect for those participants with a higher AQ than those with a lower AQ.

It should be noted that an individual's categorization as having a low or high AQ was relative to the rest of the sample, and not derived from an absolute diagnostic criterion (an AQ score in excess of 32 is indicative of clinical levels of autistic traits; Baron-Cohen et al. 2001a). However, the highest AQ score in the current sample was $25(n=2)$. The mean AQ score obtained was 15.3 ( $\mathrm{SD}=4.6$ ), while the mean score of the high AQ group was $19.1(\mathrm{SD}=2.7)$, which are very similar to scores obtained in comparable studies. Nevertheless, the prevalence of relatively low AQ scorers is a shortcoming. Future research could endeavor to target demographics posited to have a high $\mathrm{AQ}$, such as those who study mathematics and science (Baron-Cohen et al. 2001a).

This study showed that inferences about other people's dispositions are formed involuntarily from repeated exposure with minimal deliberate or explicit reasoning or attentional allocation. The ability to implicitly learn social information would reduce the cognitive effort associated with social interactions, in particular when faced with a continuous stream of complex social stimuli. These latter scenarios are especially challenging for individuals with ASD, which could partly be explained by the proposed impairments in implicit social learning. Subsequent experiments will need to address this issue in the ASD population. An implication of such a deficit in individuals with ASD may be that, in order to navigate the social world, they rely on explicit reasoning and on generalized mechanisms that are typically used for the processing of non-social stimuli. Indeed, there is evidence that whilst implicit Theory of Mind is impaired in ASD, explicit Theory of Mind remains intact (Ruffman et al. 2001). Further, atypical (non-social) strategies have been observed in individuals with ASD in the domains of action anticipation (Hudson et al. 2011), action imitation (D'Entremont and Yazbek 2007) and mental state reasoning (BaronCohen et al. 1999; Zalla et al. 2009).

\section{Conclusions}

The foremost finding of the current study was that the low AQ group showed evidence for implicit social learning whereas the high AQ group did not. The pro/antisocial intentions of another can be implicitly attributed on the basis of social cues witnessed in a previous social encounter and go on to affect one's subsequent responses to that individual, without one being aware of it. Furthermore, this effect is determined by individual differences in autistic-like traits. Those with a better ability to involuntary "pick up" the meaning of social contingencies learned that one identity held an antisocial disposition towards them, and consequently showed a smaller gaze-cueing effect in response to that individual. Those with more autistic-like traits did not pick up on such cue combinations, and their cueing effect was not affected by the identity of the agent. Differences between low and high AQ groups on several 
cognitive capacities have been reported before (e.g. BaronCohen et al. 2001b; Grinter et al. 2009; Lombardo et al. 2007), but this is the first study revealing a difference in implicit social learning. We can only speculate about the implications of our findings for individuals with ASD. In line with the theory of an autism spectrum continuum underlying the entire population (Baron-Cohen et al. 2001a), extrapolation of the results of the High AQ group to individuals with ASD would suggest more severe deficits in the ability of implicit social learning in ASD, which may well underpin core deficits in social understanding in ASD.

We do not yet know whether the higher propensity for implicit social learning in the low AQ group is specific to social learning. Subsequent studies could contrast the ability for implicit social learning with that for implicit non-social learning (e.g. learning of contingencies between motions of physical objects). As those with more autisticlike traits are thought to be more sensitive to lawful systematic contingencies, one might speculate that the high AQ group will outperform the low AQ group with respect to non-social implicit learning.

Acknowledgments This work was supported by the 80th Anniversary University of Hull Studentship Scheme and by the Netherlands Organisation for Scientific Research (NWO, No. 446-08-003).

\section{References}

Adams, R. B., \& Kleck, R. E. (2003). Perceived gaze direction and the processing of facial displays of emotion. Psychological Science, 14, 644-647.

Adams, R. B., \& Kleck, R. E. (2005). Effects of direct and averted gaze on the perception of facially communicated emotion. Emotion, 5, 3-11.

Baron-Cohen, S. (1995). Mindblindness: An essay on autism and theory of mind. Cambridge: Mass MIT Press.

Baron-Cohen, S. (2005). The empathizing system: A revision of the 1994 model of the mindreading system. In B. Ellis \& D. Bjorklund (Eds.), Origins of the social mind: evolutionary psychology and child development (pp. 468-493). New York: Guilford Press.

Baron-Cohen, S., O'Riordan, M., Stone, V., Jones, R., \& Plaisted, K. (1999). Recognition of faux pas by normally developing children and children with Asperger syndrome or high-functioning autism. Journal of Autism and Developmental Disorder, 29, 407-418.

Baron-Cohen, S., Wheelwright, S., Skinner, R., Martin, J., \& Clubley, E. (2001a). The Autism-Spectrum Quotient (AQ): Evidence from Asperger syndrome/high-functioning autism, males and females, scientists and mathematicians. Journal of Autism and Developmental Disorders, 31, 5-17.

Baron-Cohen, S., Wheelwright, S., Spong, A., Scahill, V., \& Lawson, J. (2001b). Are intuitive physics and intuitive psychology independent? Journal of Developmental and Learning Disorders, 5, 47-78.

Baron-Cohen, S., Wheelwright, S., Hill, J., Raste, Y., \& Plumb, I. (2001c). The "reading the mind in the eyes" test revised version:
A study with normal adults, and adults with Asperger syndrome or high-functioning autism. Journal of Child Psychology and Psychiatry, 42, 241-252.

Bayliss, A. P., \& Tipper, S. P. (2005). Gaze and arrow cueing of attention reveals individual differences along the autism spectrum as a function of target context. British Journal of Psychology, 96, 95-114.

Bayliss, A. P., \& Tipper, S. P. (2006). Predictive gaze cues and personality judgments: Should eye trust you? Psychological Science, 17, 514-520.

Bayliss, A. P., di Pellegrino, G., \& Tipper, S. P. (2005). Sex differences in eye gaze and symbolic cueing of attention. Quarterly Journal of Experimental Psychology Section A Human Experimental Psychology, 58, 631-650.

Bayliss, A. P., Griffiths, D., \& Tipper, S. P. (2009). Predictive gaze cues affect face evaluations: The effect of facial emotion. European Journal of Cognitive Psychology, 21, 1072-1084.

Brown, W. M., \& Moore, C. (2002). Smile asymmetries and reputation as reliable indicators of likelihood to cooperate: An evolutionary analysis. In S. P. Shohov (Ed.), Advances in psychology research (Vol. 11, pp. 19-36). Hauppauge: Nova Science Publishers.

Chawarska, K., Klin, A., \& Volkmar, F. (2003). Automatic attention cueing through eye movement in 2 -year-old children with autism. Child Development, 74, 1108-1122.

Constantino, J. N., \& Todd, R. D. (2003). Autistic traits in the general population: A twin study. Archives of General Psychiatry, 45, 719-726.

D'Entremont, B., \& Yazbek, A. (2007). Imitation of intentional and accidental actions by children with autism. Journal of Autism and Developmental Disorders, 37, 1665-1678.

Deaner, R. O., Shepherd, S. V., \& Platt, M. L. (2007). Familiarity accentuates gaze cuing in women but not men. Biology Letters, 3, 64-67.

Driver, J., Davis, G., Ricciardelli, P., Kidd, P., Maxwell, E., \& BaronCohen, S. (1999). Gaze perception triggers reflexive visuospatial orienting. Visual Cognition, 6, 509-540.

Friesen, C. K., \& Kingstone, A. (2003). Abrupt onsets and gaze direction cues trigger independent reflexive attentional effects. Cognition, 87, B1-B10.

Friesen, C. K., Ristic, J., \& Kingstone, A. (2004). Attentional effects of counterpredictive gaze and arrow cues. Journal of Experimental Psychology-Human Perception and Performance, 30, 319-329.

Frischen, A., \& Tipper, S. P. (2006). Long-term gaze cueing effects: Evidence for retrieval of prior states of attention from memory. Visual Cognition, 14, 351-364.

Golan, O., Baron-Cohen, S., Hill, J., \& Rutherford, M. D. (2007). The "reading the mind in the voice" test-revised: A study of complex emotion recognition in adults with and without autism spectrum disorder. Journal of Autism and Developmental Disorders, 37, 1096-1106.

Grinter, E. J., Maybery, M. T., Van Beek, P. L., Pellicano, E., Badcock, J. C., \& Badcock, D. R. (2009). Global visual processing and self-rated autistic like traits. Journal of Autism and Developmental Disorders, 39, 1278-1290.

Happé, F. (1999). Autism: Cognitive deficit or cognitive style? Trends in Cognitive Sciences, 3, 216-222.

Hess, U., Adams, R. B., \& Kleck, R. E. (2007). Looking at you or looking elsewhere: The influence of head orientation on the signal value of emotional facial expressions. Motivation and Emotion, 31, 137-144.

Hudson, M., \& Jellema, T. (2011). Resolving ambiguous behavioural intentions by means of involuntary prioritization of gaze processing. Emotion, 11, 681-686. 
Hudson, M., Liu, C. H., \& Jellema, T. (2009). Anticipating intentional actions: The effect of gaze direction on the judgment of head rotation. Cognition, 112, 423-434.

Hudson, M., Burnett, H. G., \& Jellema, T. (2011). Anticipation of intentional actions in high- functioning autism. Journal of Autism and Developmental Disorders,. doi:10.1007/s10803-0111410-y.

Hurst, R. M., Mitchell, J. T., Kimbrel, N. A., Kwapil, T. K., \& Nelson-Gray, R. O. (2007). Examination of the reliability and factor structure of the Autism Spectrum Quotient (AQ) in a nonclinical sample. Personality and Individual Differences, 43, 1938-1949.

Jellema, T., \& Perrett, D. I. (2002). Coding of visible and hidden objects. Attention and Performance, 19, 356-380.

Jellema, T., Lorteije, J., van Rijn, S., van t' Wout, M., de Haan, E., van Engeland, H., et al. (2009). Involuntary interpretation of social cues is compromised in autism spectrum disorders. Autism Research, 2, 192-204.

Johansson, M., Mecklinger, A., \& Treese, A. C. (2004). Recognition memory for emotional and neutral faces: An event related potential study. Journal of Cognitive Neuroscience, 16, $1840-1853$.

Jones, B. C., DeBruine, L. M., Little, A. C., Conway, C. A., \& Feinberg, D. R. (2006). Integrating gaze direction and expression in preferences for attractive faces. Psychological Science, 17, $588-591$.

Kemner, C., Schuller, A. M., \& van Engeland, H. (2006). Electrocortical reflections of face and gaze processing in children with pervasive developmental disorder. Journal of Child Psychology and Psychiatry, 47, 1063-1072.

Kuhn, G., \& Kingstone, A. (2009). Look away! Eyes and arrows engage occulomotor responses automatically. Attention Perception \& Psychophysics, 71, 314-327.

Kylliainen, A., \& Hietanen, J. K. (2004). Attention orienting by another's gaze direction in children with autism. Journal of Child Psychology and Psychiatry, 45, 435-444.

Langton, S. R. H., \& Bruce, V. (1999). Reflexive visual orienting in response to the social attention of others. Visual Cognition, 6, $541-567$.

Lieberman, M. D. (2000). Intuition: A social cognitive neuroscience approach. Psychological Bulletin, 126, 109-137.

Lieberman, M. D., Gaunt, R., Gilbert, D. T., \& Trope, Y. (2002). Reflexion and reflection: A social cognitive neuroscience approach to attributional inference. Advances in Experimental Social Psychology, 34, 199-249.

Lombardo, M. V., Barnes, J. L., Wheelwright, S. J., \& Baron-Cohen, S. (2007). Self referential cognition and empathy in autism. PLOS ONE, 2, e883

Mehu, M., Grammer, K., \& Dunbar, R. I. M. (2007a). Smiles when sharing. Evolution and Human Behavior, 28, 415-422.

Mehu, M., Little, A. C., \& Dunbar, R. I. M. (2007b). Duchenne smiles and the perception of generosity and sociability in faces. Journal of Evolutionary Psychology, 5, 133-146.

Nation, K., \& Penny, S. (2008). Sensitivity to eye gaze in autism: Is it normal? Is it automatic? Is it social? Development and Psychopathology, 20, 79-97.

Plaisted, K., O'Riordan, M., \& Baron-Cohen, S. (1998). Enhanced discrimination of novel, highly similar stimuli by adults with autism during a perceptual learning task. Journal of Child Psychology and Psychiatry, 39, 765-775.

Redcay, E. (2008). The superior temporal sulcus performs a common function for social and speech perception: Implications for the emergence of autism. Neuroscience and Biobehavioral Reviews, 32, 123-142.

Reed, P., Lowe, C., \& Everett, R. (2011). Perceptual learning and perceptual search are altered in male university students with higher Autism Quotient scores. Personality and Individual Differences, 51, 732-736.

Ristic, J., Mottron, L., Friesen, C. K., Iarocci, G., Burack, J. A., \& Kingstone, A. (2005). Eyes are special but not for everyone: The case of autism. Cognitive Brain Research, 24, 715-718.

Ruffman, T., Garnham, W., \& Rideout, P. (2001). Social understanding in autism: Eye gaze as a measure of core insights. Journal of Child Psychology and Psychiatry, 41, 1083-1094.

Sander, D., Grandjean, D., Kaiser, S., Wehrle, T., \& Scherer, K. R. (2007). Interaction effects of perceived gaze direction and dynamic facial expression: Evidence for appraisal theories of emotion. European Journal of Cognitive Psychology, 19, $470-480$.

Satpute, A. B., \& Lieberman, M. D. (2006). Integrating automatic and controlled processes into neurocognitive models of social cognition. Brain Research, 1079, 86-97.

Scharlemann, J. P. W., Eckel, C. C., Kacelnik, A., \& Wilson, R. K. (2001). The value of a smile: Game theory with a human face. Journal of Economic Psychology, 22, 617-640.

Schmidt, K. L., \& Cohn, J. F. (2001). Human facial expressions as adaptations: Evolutionary questions in facial expression research. Yearbook of Physical Anthropology, 44, 3-24.

Senju, A., Tojo, Y., Dairoku, H., \& Hasegawa, T. (2004). Reflexive orienting in response to eye gaze and an arrow in children with and without autism. Journal of Child Psychology and Psychiatry, $45,445-458$.

Senju, A., Southgate, V., White, S., \& Frith, U. (2009). Mindblind eyes: An absence of spontaneous theory of mind in Asperger syndrome. Science, 325, 883-885.

Stewart, M. E., \& Austin, E. J. (2009). The structure of the AutismSpectrum Quotient (AQ): Evidence from a student sample in Scotland. Personality and Individual Differences, 47, 224-228.

Suda, M., Takei, Y., Aoyama, Y., Narita, K., Sakurai, N., Fukuda, M., et al. (2011). Autistic traits and brain activation during face-toface conversations in typically developed adults. PLOS ONE, 6, e20021.

Sutherland, A., \& Crewther, D. P. (2010). Magnocellular visual evoked potential delay with high Autism-Spectrum Quotient yields a neural mechanism for altered perception. Brain, 133, 2089-2097.

Swettenham, J., Condie, S., Campbell, R., Milne, E., \& Coleman, M. (2003). Does the perception of moving eyes trigger reflexive visual orienting in autism? Philosophical Transactions of the Royal Society B-Biological Sciences, 358, 325-334.

Tipples, J. (2005). Orienting to eye gaze and face processing. Journal of Experimental Psychology-Human Perception and Performance, $31,843-856$.

Tipples, J. (2008). Orienting to counterpredictive gaze and arrow cues. Perception \& Psychophysics, 70, 77-87.

van Dijk, E., van Kleef, G. A., Steinel, W., \& van Beest, I. (2008). A social functional approach to emotions in bargaining: When communicating anger pays and when it backfires. Journal of Personality and Social Psychology, 94, 600-614.

Vanneste, S., Verplaetse, J., Van Hiel, A., \& Braeckman, J. (2007). Attention bias toward noncooperative people: A dot probe classification study in cheating detection. Evolution and Human Behavior, 28, 272-276.

Verplaetse, J., Vanneste, S., \& Braeckman, J. (2007). You can judge a book by its cover: The sequel. A kernel of truth in predictive cheating detection. Evolution and Human Behavior, 28, 260-271.

Vlamings, P. H. J. M., Stauder, J. E. A., van Son, I. A. M., \& Mottron, L. (2005). Atypical visual orienting to gaze- and arrow-cues in adults with high functioning autism. Journal of Autism and Developmental Disorders, 35, 267-277.

Von dem Hagen, A. H., Nummenmaa, L., Yu, R., Engell, A. D., Ewbank, M. P., \& Calder, A. J. (2011). Autism spectrum traits in 
the typical population predict structure and function in the posterior superior temporal sulcus. Cerebral Cortex, 21, 493-500.

Woodbury-Smith, M. R., Robinson, J., Wheelwright, S., \& BaronCohen, S. (2005). Screening adults for Asperger syndrome using the AQ: A preliminary study of its diagnostic validity in clinical practice. Journal of Autism and Developmental Disorders, 35, 331-335.
Yamagishi, T., Tanida, S., Mashima, R., Shimoma, E., \& Kanazawa, S. (2003). You can judge a book by its cover: Evidence that cheaters may look different from cooperators. Evolution and Human Behavior, 24, 290-301.

Zalla, T., Sav, A. M., Stopin, A., Ahade, S., \& Leboyer, M. (2009). Faux pas detection and intentional action in Asperger syndrome: A replication in a French sample. Journal of Autism and Developmental Disorders, 39, 373-382. 